\title{
Smart Wearable System to Assist Asthma Patients
}

\author{
Niranjana $\mathrm{S}^{\mathrm{a}, 1}$, Hareshaa $\mathrm{S} \mathrm{K}^{\mathrm{a}}$, Irene Zibiah Basker ${ }^{\mathrm{a}}$ and Anand $\mathrm{J}^{\mathrm{b}}$ \\ ${ }^{\mathrm{a}} U G$ Student-Department of ECE, KCG College of Technology, Chennai, India. \\ ${ }^{\mathrm{b}}$ Associate Professor-Department of ECE, KCG College of Technology, Chennai, India.
}

\begin{abstract}
Asthma is a perpetual lung infection that inflames and shortens the airways leading to intricacy in breathing. It affects 300 million human life globally out of which 20 million people are Indians, and every year about 2.5 lakh people die of asthma prematurely around the world. The estimation of asthma patients globally will exceed 100 million by the year 2025. All the deaths due to asthma are preventable with proper care and guidance [1]. This paper provides a user-friendly wearable device included with an android app that monitors the environmental factors faced by asthma patients as well as their health parameters and transmits the collected data to a cloud platform. The parameters are deliberated using special sensing element like temperature sensing unit, humidity sensing unit, gas sensor unit and pulse rate sensor unit. The evaluated parameters are the presence of polluted gas, abnormal temperature, humidity and pulse rate, and the specific location of the user. All these parameters are regularly updated in the display unit attached to the wearable device as well as in an android app, which consists of Asthma Symptom Test (AST) to predict the probability of asthma and it gives essential precautions to the patients by gathering details from the cloud which prevents them from provoking a severe attack. The proposed system was implemented and tested in real-time environment.
\end{abstract}

Keywords. Air Quality, Asthma, Cloud, Sensors, Wearable System.

\section{Introduction}

Asthma is a persistent illness that has an effect on the lungs and weakens airways through the acquisition of excessive quality mucus which results in a limited amount of oxygen delivered to vital organs. Asthmatic people often experience various symptoms that lead to a diagnosis of asthma [2]. The accurate cause of asthma is not yet known. However, studies have revealed that a mixture of genetic and environmental factors leads to enhance in asthmatics worldwide. The major causes of asthma symptoms vary from person to person. There are different types of asthma present such as primary, intermediate and critical level; this varies from person to person. The asthma indications diverge drastically from one person to another person. Even now, one information is common with asthma, that when airways experience some asthma triggers, then it gets narrow,

\footnotetext{
${ }^{1}$ Niranjana S, UG Student, Department of ECE, KCG College of Technology, Chennai, India.

E-mail: niranjana.sivam98@gmail.com.
} 
inflamed and get filled with the mucus, and excessive amounts of mucus contribute to airway contraction [3].

According to Indian doctors, there has been an increase in air sickness in children due to an increase in pollution [4]. The framework mentioned for the detection of environmental factors by constantly evaluating the air quality using various sensors and helping the patient to reduce the risk of asthma by providing a sophisticated device along with safety precautions. If the collection of air particles exceeds a certain amount, the pollutants present in the atmosphere is shown to the patient by the android app with appropriate suggestions to them. The user's health condition is regularly observed and updated in the cloud database, which helps them to take preventive measures by them immediately and able to contact the physician if the situation is an emergency. The foremost objectives of the paper are, to monitor and improve the health conditions of asthma patients, to provide preventive measures to the patients, to observe the environment of the patient constantly, to identify the quality of air and to provide a user friendly and portable device.

Remaining article is structured as follows. Existing methods is explored in section 2. Section 3 describes the system outline. Finally, working is detailed in section 4 followed with results in section 5. At last conclusion and references are given.

\section{Existing Methods}

Under this topic we introduce a summary of some significant assistance made by numerous researchers in the subject of asthma monitoring devices using embedded and IoT:

The authors in [5] proposed an m-health system which has a mobile application used for an Android platform and a WEB application. The online structures of Firebase to control asthma by providing a tool for patients to calculate the quality of airway control. This system is grounded on Asthma Control Questionnaire (ACQ), Asthma Control Test (ACT) which is the most widely used clinical practice. This contributes to selfmanagement by shaping the quality of patient management. For usability assessment in a system, maximum of the users valued it as useful and on using the system commonly to maintain the self-management of asthma.

The authors in [6] designed a transportable device that displays the environmental disclosures practiced by an asthmatic patient and directs the data to the cloud environment. A proper technique for recognition of asthma activates is proposed to assistance recognize the factors that cause asthma in a particular person. a transportable device that displays the surrounding exposed by the patients and directs the data to the cloud. These conceptions focus on measuring various gases such as $\mathrm{CO}$, smoke and contrast it with a standard air quality index (AQI) as a reference. Patients are intimated by messages when there is a rapid change in the data.

The authors in [7] examine the air quality index (AQI) and provides personalized and localized feedback to patients which to helps them improve their health conditions. This paper focuses on both indoor and outdoor air quality. This paper proposed planning for smart asthma controlling. This gathers data using Microsoft Excel and analysis information using MATLAB, thereby gives response to provide limited and personalized smart asthma observing. AQI for outdoor air quality is considered a reference for our paper. 
The authors in $[8,9]$ used machine learning for prediction for their application, the algorithm applied for forecasting asthma threat. The study is created on Peak Expiratory Flow Rates (PEFR) for some pediatric patients. PEFR outcomes are unruffled twice in a day and separated into three groups as 'Green' (normal), 'Yellow' (mild to moderate exacerbation) and 'Red' (severe exacerbation) with orientation to their finest peak flow rate. Long Short-Term Memory (LSTM) prototypical is initiate to forecast the asthma threat groups as compared to MultiNomial Logistic (MNL) regression as it integrates the increasing properties of PM attentions. This method could possibly play an innovative part for the technical data-driven medical choice creation.

The authors in [10] proposed a structure for examining and calculating asthma exacerbation based on e-health and using medical IoT devices to gather and investigate data in physical period to regulate the harshness of asthma and to give defensive actions and measures in order to constantly display the sickness and stop the risk of asthma attack. The device provides altering messages in critical situation.

The authors in [11] offered a scheme that will be able to forecast likely asthma spell for the patients and aware them. The scheme is consisting of air pollutant nursing device joint with an Android application which helps to decrease asthma attacks for asthma patients by supervised learning technique and examining air pollutant data. Also examining modified data of patients it will be likely to recommend a new operator about the safe and unsafe region of the city. As a by-product, it will be likely to generate a high-density air pollution chart of cities to television air pollution.

The authors in [12] proposed a software and hardware system for heart beat related biomedical parameters monitoring scheme using the IoT and Sensors using ESP8266 Wi-Fi module. The system monitors and obtain the heart rate in bpm and temperate value which are observed continuously. The system is connected to the cloud and an app to convey the vital parameters of human body using the Wi-Fi module.

The author in [13] proposed the software and hardware construction of the e-health smart pulse oximeter scheme combined into Seat Display Unit (SDU) for passenger's scrutiny in-flight. The idea of this smart scheme come emerged from the information that during the flight, the inside cab conditions, such as little pressure, absence of oxygen and little humidity are threat factors in patient's misery from pulmonary illnesses, cardiovascular illnesses, venous thrombosis illnesses, asthma sicknesses, lung emphysema illnesses, surgical operations suffered recently, epilepsy, diabetes, mental illnesses and infectious illnesses. The system monitors oxygen stages in the blood and pulse amount throughout the phases such as takeoff/flight/landing to offer info on the health status of passengers and this data could be recycled in the medical field to deliver proposal to patients who have exact ailments and to travel by air. Few optimization logics which are used in biomedical applications are discussed in [14,15].

\section{System Outline}

In the proposed method consists of both hardware and software. The hardware consists of a micro-controller, gas sensing unit, temperature sensing unit, humidity sensing unit, GPS module, and pulse rate sensing unit. The sensors are attached the portable device to examine the pollutants such as carbon monoxide and carbon dioxide in the environment, to detect the rapid changes in the temperature and humidity, and to scrutinize the health 
parameters. The data found from the different sensors are delivered to the cloud intermittently. Data found in the cloud is used to investigate and offer patients with security actions through the mobile app attached to the cloud.

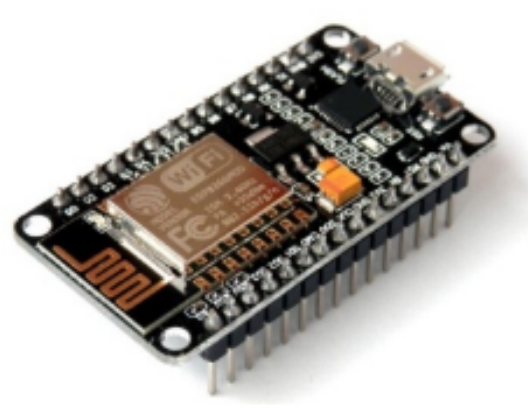

Figure 1. NodeMCU

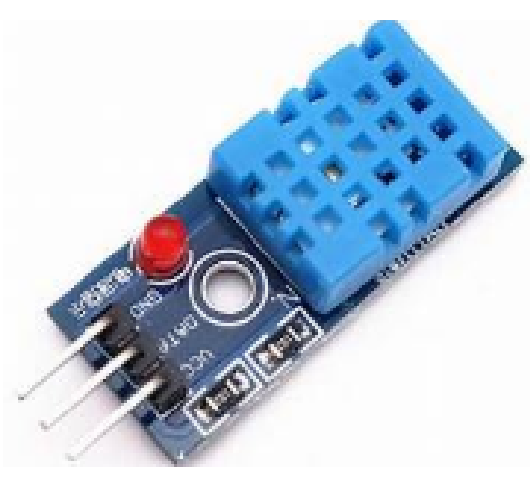

Figure 3. DHT11

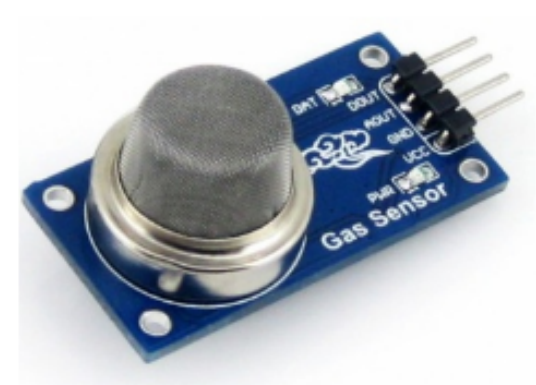

Figure 2. Gas Sensor

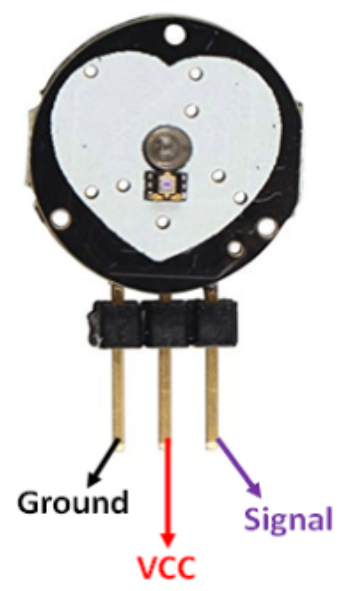

Figure 4. Pulse Sensor

\subsection{Microcontroller}

NodeMCU is a computing expansion board based on the ESP8266 integrated Wi-Fi unit. It has FCC certified Wi-Fi modules and a PCB antenna. The board incorporates over-all function General Purpose Input Output (GPIO), Inter Integrated Circuit (I2C), Analog to Digital Converter (ADC). Its firmware executed on Express ESP8266 Wi-Fi System on Chip (SoC) system. NodeMCU on average has $128 \mathrm{~KB}$ of memory. It has easy and visual scripting language. The board consists of a micro-USB port for power and communication with the Personal Computer (PC). It can be effortlessly operated from a USB to a micro USB port via a power bank. Due to the relative ease of use and the built-in Wi-Fi modules, NodeMCU is commonly preferred over other micro-controllers. 


\subsection{Gas Sensor}

The MQ-2 gas sensor is generally a susceptible sensor for identify $\mathrm{SnO} 2$ and CO. It is appropriate for escalating carbon monoxide concentrations ( $\mathrm{ppm}$ ) in polluted air. It is quick, consistent, and enduring. SnO2 is the substantial component and this sensor unit that detects gas concentrations efficiently and precisely. SnO2 has low concentrations in air. It can regularly compute $\mathrm{CO}$ concentrations from 20 to $2000 \mathrm{ppm}$. The output of the sensor specifies analog resistance. The conductor circuit is effortless and consists of a voltage divider that operates from the heater coil with $5 \mathrm{~V}$ AC or DC, which is connected to load resistance and output ADC or linear.

\subsection{Humidityand Temperature sensor}

Here we are using cost-effective DHT11, digital temperature and humidity sensor unit. It has the feature of a humidity sensor and a thermistor unit that estimates the nearby atmosphere and provides a digital indication on the data pin. It is handy but requires more time to position the data. The output is obtained within 2 seconds.

\subsection{Heart rate sensor}

The heart rate sensing unit which is used sense the pulse amount of the user. The analog output is acquired by placing the finger on the LED. It gives output in BPM and it can also be used to find the oxygen level in the blood. It can be easily integrated with any micro-controller. It also includes an examining app that regularly monitors e data and updates in the app. It is a painless method to compute the heart rate in an inexpensive manner.

\section{5. $O L E D$}

OLED (Organic Light-Emitting Diodes) is made by keeping the organic thin films between two conductors in a series manner. A bright light is produced when the current is applied to it. OLEDs are more proficient than LEDs as they are thinner in a structure that does not need a backlight. It is not only efficient but also provides good image quality and this can be made more flexible and transparent.

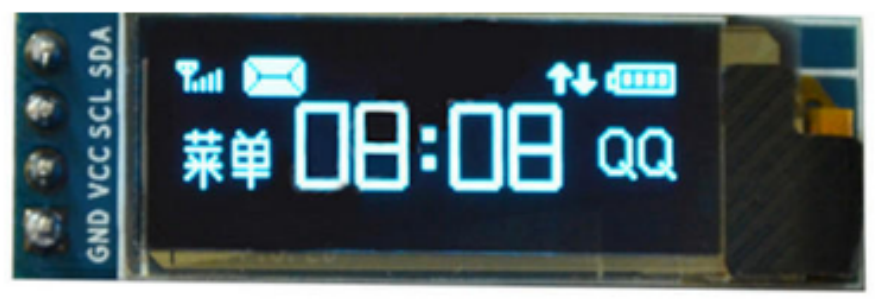

Figure 5. OLED 


\subsection{Arduino Development Environment}

The Arduino IDE which is abbreviated as Arduino Integrated Development Environment is a software which is applied to write and upload code into a dissimilar Arduino boards. This software consists of various in-built libraries that enclose essential functions which makes programming easy. It also has a immense characteristic which marks it opportune to clear up mistakes and troubleshoot problems. The NodeMCU can also be planned using Arduino IDE by connecting the libraries essential and selecting ESP8266 NodeMCU in the Board Manager Option. The NodeMCU microcontroller is programmed using embedded $\mathrm{C}$ language.

\subsection{Firebase}

Firebase is a cloud platform that is used to gather data in real time. Firebase the whole thing with the platforms like Arduino, ESP8266 and Raspberry Pi. It has a commanding API. It allows the user to send and observe the data in cloud. If the threshold from the data is found correctly, there are alternatives to set triggers. Data are immediately reorganized to the cloud for each 1 second, permitting real-time data observing. The dashboard in IO allows the user to allow feeds in different formats of his choice. Data acknowledged from the gas sensor is broadcasted to this cloud platform.

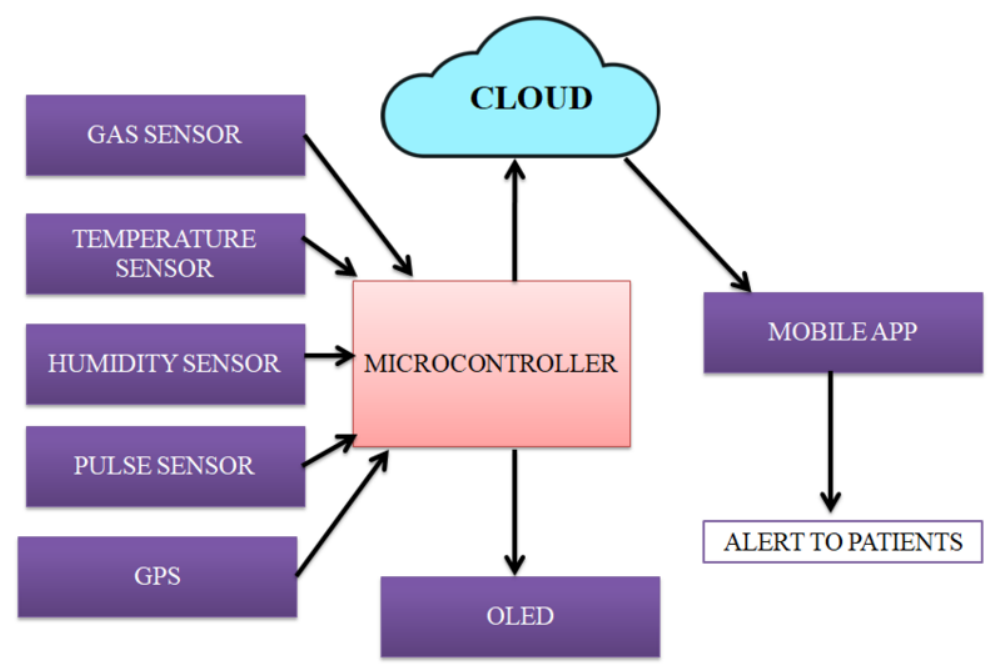

Figure 6. Basic Block Diagram of Prototype

\section{Working}

Our idea aims to decrease the risk of asthma by providing a compact and transportable device incorporated with an android app which gives continuous precautions to the patients whenever needed. The hardware consists of various sensors such as gas sensor which detects the hazardous gases in the atmosphere, a temperature sensor to intellect 
the temperature, a humidity sensor to intellect the changes in the climate, a pulse sensor which detects the heart rate as well as the oxygen level in the blood, a GPS module to track the user's location and also it identifies the polluted area and this details are updated to the government as healthy as to the cloud and a $\mathrm{Wi}-\mathrm{Fi}$ module is provided to update the responses of the sensor to the cloud. All the details from these sensors are uploaded to the cloud, and frequent changes in these responses are changed to the patients via an android app. The superior step in the android app is to register the personal details of the user, and then the system continues with an asthma symptom test which contains a number of questions to test the symptoms of asthma before consulting a doctor. If the test comes out to positive, then two selections are provided to consult the doctor or to take the test again. The consistent precautions are rapidly given to the registered user by accurately comparing their health conditions as well as environmental conditions. The user's health condition is regularly watched and restructured in the database or the cloud for succeeding reference. In the case of a critical situation, the app will use its superior feature to call the doctor or a registered member. The device is simple and wearable that is used by asthmatic patients and even it can be used by the ordinary person. This app is accessible and inspires the user to care for their health and yield the precautions in stark conditions which initially make them self-dependent and the patients will be able to therapy before having an asthma attack.

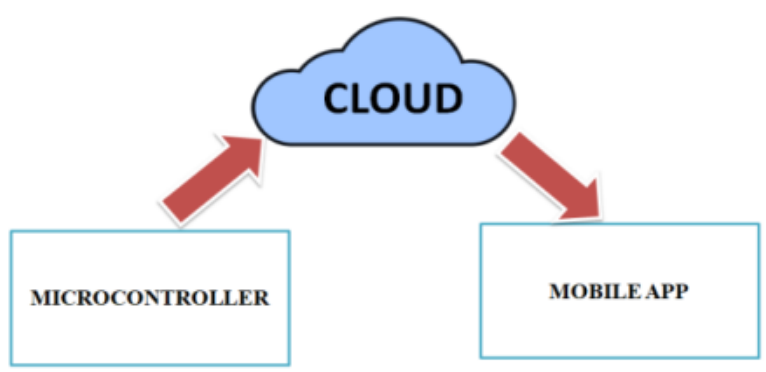

Figure 7. Basic Overview

\section{Results and Discussion}

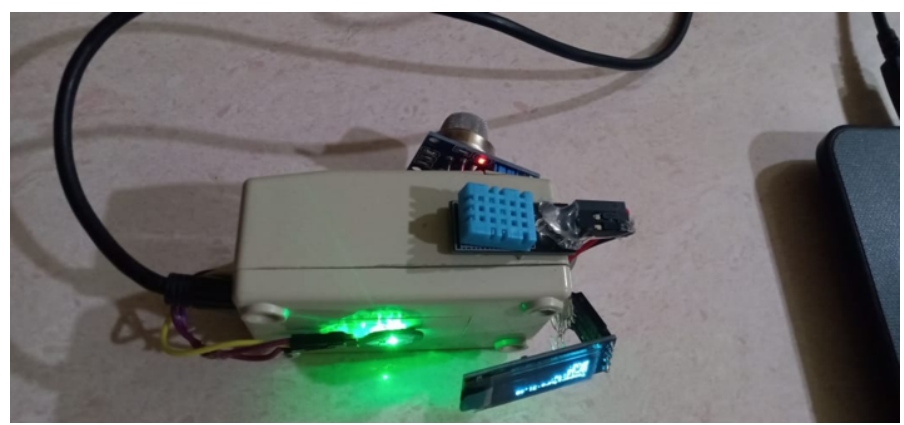

Figure 8. Basic Prototype 
Figure 8 is the basic prototype of our paper which consists of DHT11 (temperature and humidity sensor), pulse rate sensor, gas sensor and OLED. This hardware setup is integrated with the cloud and mobile app.

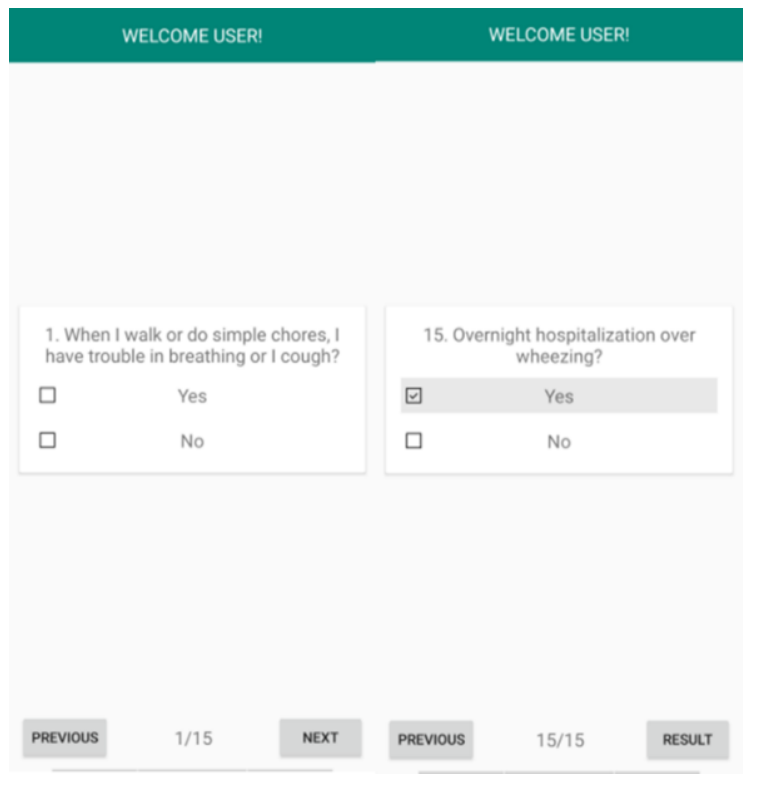

Figure 9. Asthma Symptom test

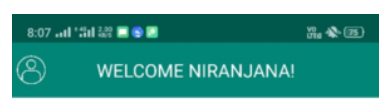

You have $66 \%$ probability of getting/having Take up the test again to review your health. Note: Carry inhaler with you. Drink hot water.
Avoid cold food, ice creams, cakes, cheese and such item.

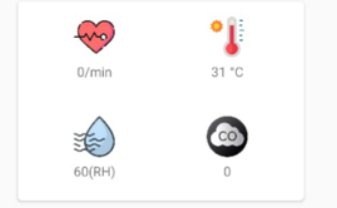

Current Location:

Latitude: 10.2889075 Longitude: 77.9194237 CONSULT DOCTOR TAKE UP THE TEST

Figure 10. Android app

Figure 9 is the second section of Android app, is the beginning of the asthma symptom test (AST).AST is few Yes or No questions which is used to predict the probability of asthma. In the Figure 9 is the first question of AST and is the last question of AST respectively. The probability of asthma is predicted by the number of Yes or No questions answered by the user. Figure 10 is the user output of Android app which provides the probability of asthma; precautions need to be taken which is provided according with probability of asthma and environmental conditions around the patients. Location of the patients is also displayed. In case of emergency situation, the user can consult doctor and share his location for help.

\section{Conclusion}

The projected system consists of hardware as well as software components. The hardware components are banged to develop a dense and transportable device made up of various sensors such as gas sensor, pulse rate sensor and temperature and humidity sensor and also an OLED is used for the display of these values rapidly. The transportable device is impeccably integrated with an android app. The sensor's response is regularly updated to the cloud and this is used to give precautions to the user for the quick changes in the sensor response with the help of online sorts of the firebase cloud. These suitable precautions are delivered in the inventive app along with the present data of the sensor from the cloud. The future scope of the system is to implement machine learning in the 
system to track the user and also to involve a spirometer test that will be joined with the app as well as device.

\section{References}

[1] The Global Asthma Report 2014. Auckland. New Zealand: Global Asthma Network. 2014.

[2] Hwang L. "Global Strategy for Asthma Management and Prevention". Global Initiation for Asthma 2018 (Vol. 112, No. 3, p. 8).

[3] Cottini M, Asero R. Asthma phenotypes today. Eur Ann Allergy Clin Immunol. 2013 Feb 1;45(1):17-24.

[4] Air Pollution and Health in India: A review of the current evidence and opportunities for the future July 2017. Public Health Foundation of India/Centre for environmental Health, India. 2017:1-64.

[5] Silva TA, Costa MG, Stelmach R, Bley PK, Gutierrez MA, Costa Filho CF. Development of a system mobile-based to assist asthma self-management. In2018 3rd Biennial South African Biomedical Engineering Conference (SAIBMEC) 2018 Apr 4 (pp. 1-4). IEEE.

[6] Indulakshmi S, Adithya M, Anirudh AR, Jawahar A. Design and Development of Prototype Model for Asthma Trigger Detection. In2018 International Conference on Wireless Communications, Signal Processing and Networking (WiSPNET) 2018 Mar 22 (pp. 1-5). IEEE.

[7] Isaac N, Sampath N, Gay V. SAM smart asthma monitoring: focus on air quality data and Internet of Things (IoT). In2018 12th International Symposium on Medical Information and Communication Technology (ISMICT) 2018 Mar 26 (pp. 1-6). IEEE.

[8] Kim D, Cho S, Tamil L, Song DJ, Seo S. Predicting asthma attacks: effects of indoor PM concentrations on peak expiratory flow rates of asthmatic children. IEEE Access. 2019 Dec 18;8:8791-7.

[9] Luo Y. Environmental cost control of coal industry based on cloud computing and machine learning. Arabian Journal of Geosciences. 2021 Jun;14(12):1-6.

[10] Maach A, El Alami J. A fog-driven IoT e-Health framework to monitor and control asthma exacerbation. In2019 International Conference on Wireless Networks and Mobile Communications (WINCOM) 2019 Oct (pp. 1-6). IEEE.

[11] Hoq MN, Alam R, Amin A. Prediction of possible asthma attack from air pollutants: Towards a high density air pollution map for smart cities to improve living. In2019 International Conference on Electrical, Computer and Communication Engineering (ECCE) 2019 Feb 7 (pp. 1-5). IEEE.

[12] Ajay H, Rao AR, Balavanan M, Lalit R. A Novel Cardiac Arrest Alerting System using IoT. International Journal of Science Technology \& Engineering. 2017;3(10):78-83.

[13] AILENI RM, PAŞCA S, FLORESCU A. E-health monitoring by smart pulse oximeter systems integrated in SDU. In2019 11th International Symposium on Advanced Topics in Electrical Engineering (ATEE) 2019 Mar 28 (pp. 1-4). IEEE.

[14] Anand J, Rajaa Paul Perinbam J, Meganathan D. Design of GA-based Routing in Biomedical Wireless Sensor Networks. International Journal of Applied Engineering Research (IJAER). 2015;10(4):9281-92.

[15] Anand J, Perinbam JR, Meganathan D. Q-learning-based optimized routing in biomedical wireless sensor networks. IETE Journal of Research. 2017 Jan 2;63(1):89-97. 